Pacific Northwest

National Laboratory

Operated by Battelle for the

U.S. Department of Energy

\section{Adaptive Management Platform for Natural Resources in the Columbia River Basin}

\author{
L.W. Vail \\ R.L. Skaggs
}

May 2002

Prepared for the U.S. Department of Energy under Contract DE-AC06-76RL01830 


\title{
DISCLAIMER
}

This report was prepared as an account of work sponsored by an agency of the United States Government. Neither the United States Government nor any agency thereof, nor Battelle Memorial Institute, nor any of their employees, makes any warranty, express or implied, or assumes any legal liability or responsibility for the accuracy, completeness, or usefulness of any information, apparatus, product, or process disclosed, or represents that its use would not infringe privately owned rights. Reference herein to any specific commercial product, process, or service by trade name, trademark, manufacturer, or otherwise does not necessarily constitute or imply its endorsement, recommendation, or favoring by the United States Government or any agency thereof, or Battelle Memorial Institute. The views and opinions of authors expressed herein do not necessarily state or reflect those of the United States Government or any agency thereof.

\author{
PACIFIC NORTHWEST NATIONAL LABORATORY \\ operated by \\ BATTELLE \\ for the \\ UNITED STATES DEPARTMENT OF ENERGY \\ under Contract DE-AC06-76RL01830
}

Printed in the United States of America

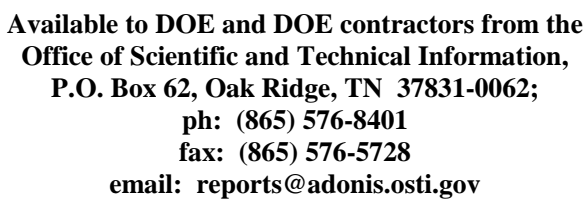

Available to the public from the National Technical Information Service, U.S. Department of Commerce, 5285 Port Royal Rd., Springfield, VA 22161 ph: (800) 553-6847 fax: $(703) 605-6900$

email: orders@ntis.fedworld.gov

online ordering: http://www.ntis.gov/ordering.htm 
PNNL-13875

\section{Adaptive Management Platform for Natural Resources in the Columbia River Basin}

Lance W. Vail

Richard L. Skaggs

May 2002

Prepared for the U.S. Department of Energy

under Contract DE-AC06-76RL01830 


\section{Abstract}

This document describes a conceptual framework to operationalize adaptive management.

Adaptive management is a systematic and rigorous scientifically defensible program of learning from the outcomes of management actions, accommodating change, and improving management. The framework, referred to as the Adaptive Management Platform (AMP), would provide significant improvements in their natural resource and fish and wildlife decisions in the very near term. The platform is a design that connects various modules to ensure that the decision-making needs are met. The modules are data management, visualization tools, optimization algorithms, and models of physical and biological processes. When fully developed, AMP will enable planners and responsible entities to collectively and continuously integrate data and decisions, assess cumulative trends and outcomes over time, and demonstrate discipline and accountability. AMP would function at the basin, ecoprovince, as well as the subbasin scale. AMP would assist in ensuring that uncertainties from individual modules and analytical integration are properly presented to decision makers.

\section{Objectives}

The Adaptive Management Platform (AMP) will provide the ability to defensibly, consistently and rapidly evaluate restoration policy and specific strategies at the subbasin scale relative to a basin-wide vision. The platform will fully exploit available data, information and analytical methods (e.g., models) to establish comprehensive, science-based tradeoff relationships between various alternatives.

This platform will support planners in making and defending decisions while simultaneously enabling stakeholders to understand and participate in the decision-making process. The platform overcomes limitations of current practices by enabling planners to:

- Quantify real and expected productivity improvements from existing and planned projects,

- Understand and communicate tradeoffs between different alternatives,

- Assess relative value of additional information,

- Utilize dramatically increasing volumes of data, and

- Utilize advanced models.

In the near-term this project will provide guidance and information tools (models, etc.) to support the ongoing subbasin planning process. These guidance documents and tools will not be prescriptive, but supportive to the stated needs of planners.

By providing the necessary information infrastructure in a nonproprietary platform, AMP will allow environmental planning to: start much earlier, be performed for significantly lower cost, 
consider and analyze cost and benefits of alternatives more completely, utilize advanced models and data, and advance more rapidly toward implementation.

Significant improvements in Accountability, Accessibility, and Adaptability were identified as three critical requirements of AMP. To provide these requirements, three integrated toolboxes (decision toolbox, model toolbox, and data toolbox) are required. Toolboxes will include both software and hardware.

\subsection{Need}

Major challenges currently facing the Columbia River Basin are management of over-prescribed water resources, Endangered Species Act (ESA)-driven requirements for salmonids and other species, and Western states power demands that place critical demands on the regional hydrosystem. Looking more closely at only a single dimension of these challenges, the Bonneville Power Administration (BPA), along with other Federal agencies, is required by the ESA to protect and foster recovery of salmon and steelhead in the Columbia River Basin. The Federal Caucus' Draft Basin-wide Salmon Recovery Plan, among its recommendations, calls for Federal Columbia River Power System (FCRPS) operations to employ aggressive flow and spill management measures to achieve rigorous salmonid survival goals over discrete time intervals. The National Marine Fisheries Service's (NMFS) Biological Opinion on "Reinitiation of Consultation on Operation of the Federal Columbia River Power System" (December 2000) places even further demands on FCRPS operations for salmon recovery. 


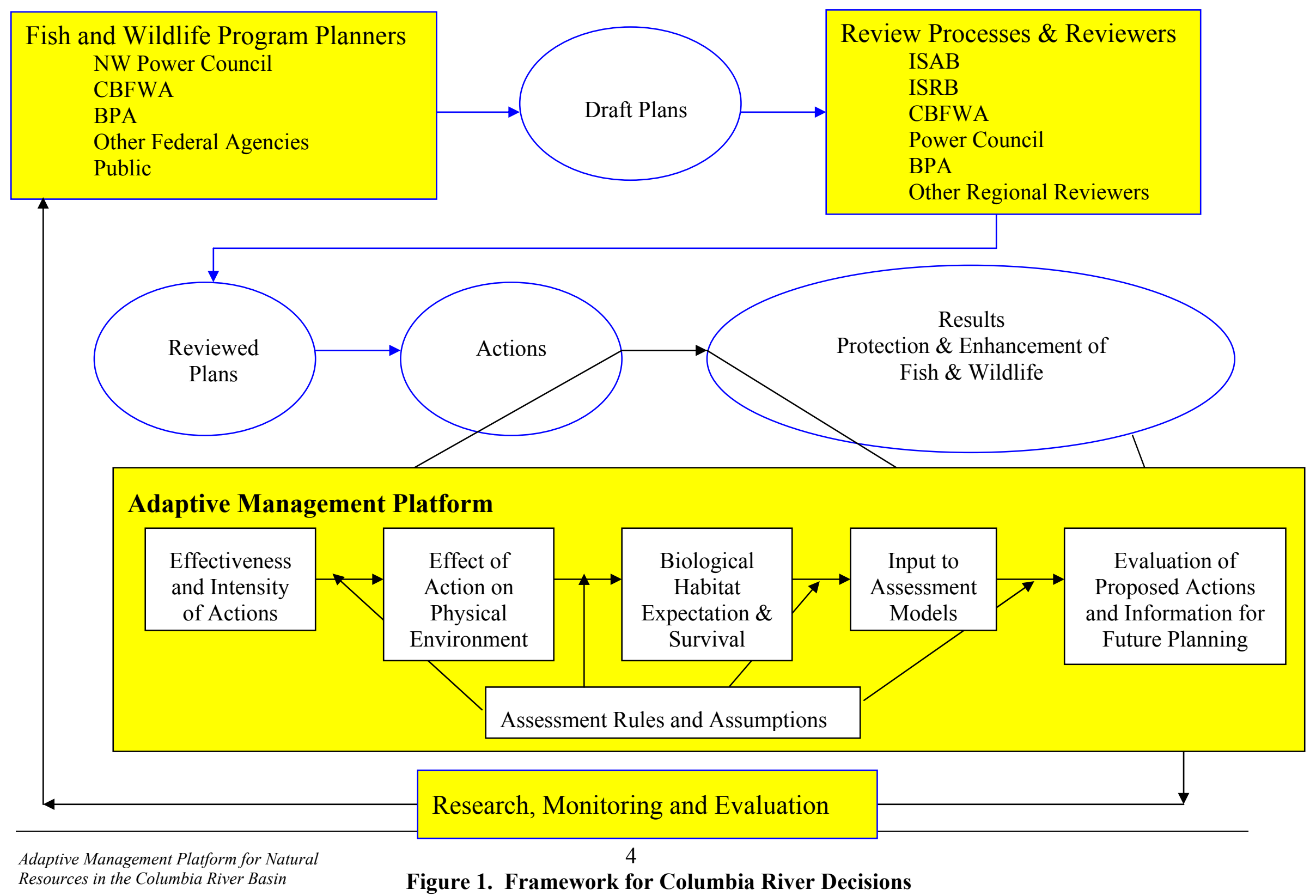


The costs during the period 1997 - 2001 for fish and wildlife measures, revenue losses from reduced power production, and costs of replacement power are estimated at approximately $\$ 430$ million annually. In the face of the recent drought and soaring energy costs, the costs for flow and spill measures, if water were available to accommodate them, would increase by order-ofmagnitude levels. Even with conservative assumptions of normal climatic and hydrologic conditions over the next decade, Northwest regional taxpayers, stakeholders, and the Federal government will invest billions of dollars in salmon recovery. Significant uncertainties associated with the benefits of these investments are further exacerbated by uncertainties in power demand, hydro potential, volatile power market prices, nuclear plant performance, natural gas price variability, etc. Given the regional and financial scale of these issues, there is growing insistence that investment of these funds should be based upon whether demonstrable, measurable, cost effective progress is being made on all elements of the program.

\subsection{Background}

Natural resource managers are constantly required to balance multiple, conflicting, incommensurate objectives in an environment characterized by high levels of uncertainty, varying data quality and availability, and competing models and approaches. The reliability of natural resources management policy and decisions depends on the ability of measurements, response models, process models, and policy models to interact with each other across the variety of temporal and spatial scales each represents. It also requires a cautious, probing, adaptive approach founded on fundamental economic principles, the success of which depends upon improved understanding, predictive accuracy, and iterative performance assessment. This is adaptive management.

Given the uncertainties in ecosystem restoration and the experimental nature of many restoration projects, the principles of adaptive management can be useful both in planning and long-term implementation of restoration projects. Adaptive management is a systematic and rigorous scientifically defensible program of learning from the outcomes of management actions, accommodating change, and improving management. While generally advocated, adaptive management has proven difficult to implement due to: 1) its direct and indirect costs (e.g., the costs of improved understanding through research and the political risks of potentially having clearly identified failures), and 2) the lack of readily available, widely implemented tools for resolving critical natural resource management issues (scientific methods for analyzing, understanding, and managing problems within complex and anthropogenically altered environmental systems).

Phenomenal advances in computational and data management resources, the proliferation and acceptance of the Internet, and continued evolution of science-based process models have outpaced current public institutions. Significant improvements in the manner that natural resources decisions are made are possible in the very near term. However, to realize the benefits of these improvements, an analytical framework or platform is required to operationalize adaptive management. The platform would connect various modules to ensure that the decisionmaking needs are met. The modules are data management, visualization tools, optimization algorithms, and physical, biological and analytical models. The platform must ensure that discrepancies in the fundamental spatial and temporal scales of the respective modules are 
managed properly. Specifically the platform must function at the basin and ecoprovince, as well as the subbasin scale. The platform must assist in ensuring that uncertainties from individual modules and the integrated analysis are properly represented to the decision maker.

Like the Internet, the platform (i.e., AMP) itself is not something that someone owns. However, it will allow the use of models or data that are proprietary. It is an agreement or protocol formalized by an accessible software and hardware infrastructure that ensures proper communication between modules. Like the Internet, AMP will establish (and fulfill) a new expectation of accountability, accessibility, and adaptability:

- Accountability - The primary goal of AMP is to support making decisions. To be useful, it must ensure that the tradeoffs between multiple objectives (of interest to both the decision maker and to stakeholders) are clearly articulated. AMP should also communicate the sources, magnitude, and costs of key uncertainties.

- Accessibility - AMP will provide rapid access to models, data, and the rationale that underpin decisions. These are growing requirements of natural resource management. The ability to drill-down through data and models ensures that decision makers are less likely to repeat similar decision errors.

- Adaptability - AMP must be able to rapidly assimilate new data and models to ensure that decisions are up-to-date with the most current information. Specific tools to automate the calibration process and to assimilate real-time data are requirements of such a framework.

When deployed the platform must:

- Be decision-focused,

- Encompass basin, ecoprovince, and subbasin scales,

- Reflect integrated effects of management alternatives and natural variability on habitat conditions over long time-periods (e.g., climate variability),

- Address species of concern,

- Allow economic values to be placed on alternatives under consideration,

- Be widely accessible and facilitate regional collaboration, and

- Provide the foundation for sustainable adaptive management practices across the Basin.

\subsection{Columbia Basin Subbasin Planning}

The Northwest Power Planning Council (Council) has a long history of providing a framework in which a Fish and Wildlife Program can be developed and implemented. The Council was 
created in 1980 by the U.S. Congress to include Idaho, Montana, Oregon and Washington in regional plans for its energy needs, while at the same time mitigating the effects of the hydropower system on fish and wildlife in the Columbia River Basin. The Council is responsible for developing a program to protect, mitigate and enhance fish and wildlife of the Columbia River Basin and make annual funding recommendations to BPA for projects to implement the program.

Each year the Council reviews proposals for on-the-ground projects and research to implement the program. Projects developed during the 1980s and early 1990s were criticized for being uncoordinated activities without an underlying scientific focus. Even though the Program was founded on an adaptive management philosophy, these criticisms often prevailed. In response to these criticisms, different review processes and independent scientific panels were established. They recommended changes that the Council could use to provide a more explicit scientific foundation for program planning. The Council's Fish and Wildlife program has always been sensitive and responsive to the needs of the Region's co-managers and other agencies that have mission requirements related to Columbia River Basin.

Today, the planning process has been complicated by other needs facing the region. There are now four species and more than ten evolutionarily significant units (ESU) in the Columbia River Basin that are protected under the Endangered Species Act (ESA). The Biological Opinion (Reinitiation of Consultation on Operation of the Federal Columbia River Power System, December 2000) includes requirements for the Action Agencies (BPA, USACE, BoR) to develop implementation plans to meet the goals of the Opinion. During 1999, the NMFS initiated the formation of Technical Recovery Teams to develop recovery goals for listed salmonids in the Columbia Basin. These examples are only a few of the requirements that need to be met for successful planning of the Council's Fish and Wildlife Program.

During 1998, the Multi-Species Framework (MSF) was initiated to further the planning process. The MSF (http://www.nwframework.org/overview.html) linked "Columbia Basin fish and wildlife restoration policy to a basin-wide vision, based on a scientific foundation that recognizes that the river and its species are interrelated parts of a whole." MSF was developed to provide system-wide direction and specific strategies for fish and wildlife recovery, and objectives by which results can be evaluated.

During 2000, the Council adopted the "subbasin planning process" as the means to develop a scientifically based Columbia Basin Fish and Wildlife Program with clear vision, goals, objectives and strategies. An outline of the early steps in the subbasin planning process is presented in Figure 2, as illustrated in the NWPPC's June 6, 2000 draft of the Subbasin Planning Template. The subbasin plans produced from the process are expected to guide the review, selection, and funding for fish and wildlife recovery efforts in the Basin. The plans will be locally developed with extensive public involvement, and will be required to address the requirements of the ESA, the Clean Water Act, the Northwest Power Act, and the policies of the states and Indian tribes within the Basin. Additionally during 2000, the Council created a Regional Assessment Advisory Committee (RAAC). RAAC will provide a scientific foundation for the subbasin planning. The Council has determined that they will use the Ecosystem Diagnostic Treatment (EDT) code as the initial decision support tool for subbasin plans. 
Subbasin Assessment

$\underline{\text { Population Data }}$

Compile Data on Population Characteristics
Habitat/Ecological Process Data

Compile Data on Subbasin

Processes, Habitat and Human Inpacts

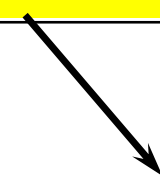

Determine Relationship between Physical Attributes and Population Data

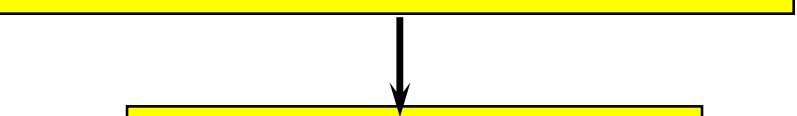

Develop Habitat Description of Key Locations for the Focal Species

Areas of Key Importance

for Focal Species

Subbasin Planning

Prioritize Watersheds for On-the-Ground

Watershed Assessment

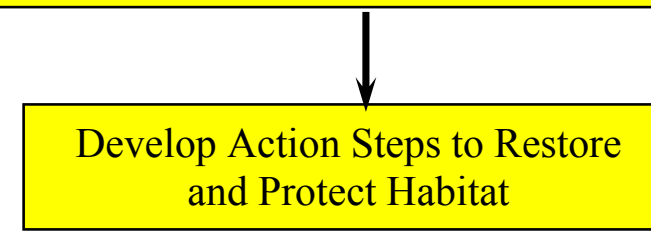

Figure 2. Synthesis of Subbasin Assessment Data 
The subbasin plans are expected to serve as an integrated compilation of all the existing information related to fish and wildlife activities in each subbasin. They will identify the goals for fish and wildlife habitat, define the objectives that will serve as measures of progress toward the goals, and establish the strategies for achieving the objectives. The subbasin-specific plans will consist of three main elements. An inventory will be developed for existing programs, activities and management plans within the subbasin. A technical assessment will be conducted to determine the biological potential of the subbasin, and the opportunities for restoration. The subbasin plans will include a management plan outlining the subbasin vision; biological objectives; strategies considering economic, legal, and environmental issues; and short- and long-term budget requirements for implementation.

The AMP will provide the subbasin planning information infrastructure required to support the science within an operational system that the region's planners can easily access and use.

\section{Adaptive Management Platform}

The Adaptive Management Platform (AMP) will support planners in making and defending their decision while simultaneously enabling stakeholders to understand and participate in the decision-making process. Planners are currently limited in their ability to:

- Understand, visualize, and communicate tradeoffs between different alternatives,

- Utilize and manage dramatically increasing volumes of data,

- Utilize and continuously refine advanced models.

By providing the necessary information infrastructure in a nonproprietary framework, AMP will improve the environmental planning process by allowing environmental planning to be started much earlier, be performed for significantly lower cost, consider alternatives more completely, and utilize advanced models and data. 


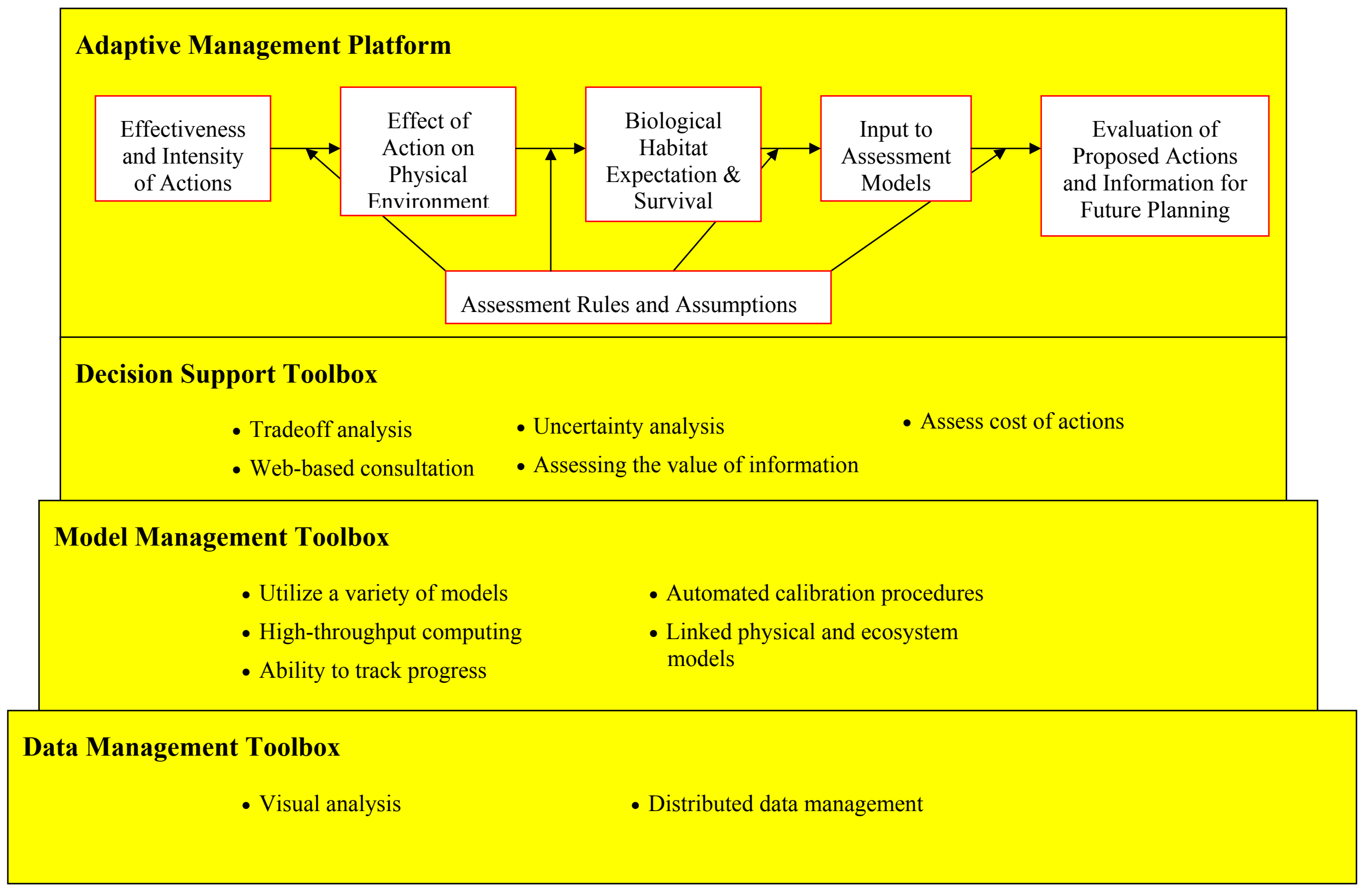

Figure 3. Tools and Methods for Decisions and Plans 


\subsection{Critical Requirements}

AMP is a system designed to integrate data, models and tools in order to advance the effectiveness of natural resource decision-making. Three key themes have driven AMP development (accountability, accessibility, adaptability).

\subsubsection{Accountability}

- AMP will clearly articulate the tradeoffs between multiple objectives.

- AMP will communicate the likelihood of making decision errors that result from model and data uncertainty.

- AMP will create a bias towards robust alternatives.

- AMP will facilitate the sharing of process knowledge.

- AMP will show the models, data, and assumptions used to reach decisions.

\subsubsection{Articulating tradeoffs}

Environmental planning decisions must consider multiple objectives, some of which conflict. Many objectives cannot be easily measured in a common way (such as dollars). The relative value of one objective to another is a policy decision that must be left to policy makers to decide. AMP encourages users to evaluate impacts using metrics that fit each resource (acres, miles, dollars) to more fully appreciate complex, non-linear relationships.

Environmental planning decisions may have unintended adverse consequences to unforeseen aspects of the environment. For instance, buying water rights to increase instream flows may have an adverse economic impact on local economies, lower-income farmer workers, and longer term water access. To reduce the likelihood that such impacts could occur without sufficient warning, AMP will support as many objectives as are required for any analysis. By adding a social-political-economic module to AMP, the impacts of leasing, retention of rights, and local impacts can be considered and weighed.

The Tradeoff Tool in the Decision Toolbox will articulate tradeoffs. The Tradeoff Tool will allow users to select any combination of objectives for axes. The Tradeoff Tool will also allow users to eliminate inferior alternatives.

\subsubsection{Communicating uncertainty}

An important need for advancing science-based natural resource management is developing practical procedures for considering uncertainty in decision-making. Making tradeoffs among multiple conflicting objectives is complicated by numerous sources of uncertainty that make predictions imprecise. As illustrated by conflicts over fish recovery, it is difficult to avoid stalemates in decision-making because tradeoff reliability can always be disputed. Identification 
of key uncertainties allows scientists, decision makers, and stakeholders to more effectively focus data gathering and analysis on variables that have the greatest influence on the target objectives or issues.

The Tradeoff Tool will aid communication of uncertainty by visually representing a cluster of equally feasible alternative outcomes based on a variety of assumptions. Using tools in the Model Toolbox, users will find patterns that identify additional data that are most likely to resolve uncertainty issues.

\subsubsection{Sharing process knowledge}

While data are generally site-specific, process knowledge is transferable to other locations. The analytical process developed at one location can often be transferred to another location given adequate similarity. For instance, sediment generation estimation methods generated from one location can be applied to other locations with similar characteristics. AMP encourages sharing by making process knowledge and data more readily available. Sharing knowledge ensures a common basis for comparing strategies since the same approach is used for different regions. This avoids biasing resource allocation prioritization efforts due to using different tools in different locations.

The Model Toolbox and Data Toolbox will support sharing by providing a reliable way of archiving and retrieving data and models.

\subsubsection{Creating robust alternatives}

Some level of uncertainty is inevitable. A robust alternative reflects the desire to manage risk by balancing caution and probing — caution to avoid adverse outcomes — probing to achieve good outcomes. An alternative may have a very good anticipated outcome based on a certain set of assumptions. However, if the same alternative results in poorer outcomes based on equallyfeasible assumptions, the alternative is not very robust. The willingness to forgo the opportunity for a very good outcome to avoid the risk of a poorer outcome demonstrates the value of a robust alternative.

The Tradeoff Tool in the Decision Toolbox will provide pattern recognition tools for highlighting alternatives more that are more robust.

\subsubsection{Opening the black box}

Accountability includes clear documentation of models and data. The Data Toolbox will capture data about models (e.g., purpose, algorithms, ownership, data created, and input parameters). Integrated into AMP is the drill-down tool. This tool will offer a guided tour of the development of an outcome. By clicking on a point in the tradeoff charts and the drill-down tool will show the models, key assumptions, and data that went into arriving at that outcome. The drill-down tool opens up the black box that decision-makers often must contend with when relying on sophisticated models and data sets. The tool allows users to quickly focus on points of contention and conduct sensitivity analyses to resolve uncertainties. 


\subsubsection{Accessibility}

- AMP will provide reliable and rapid access to models, data, and the rationale that underpin decisions.

- AMP will provide the ability to "drill-down" through decisions, data and models.

- AMP will ensure maximum access by being Web-accessible.

- AMP will provide safe collaboration for the decision-making process.

\subsubsection{Access to models, data, and decisions}

AMP will provide reliable and rapid access to models, data, and the rationale that underpin decisions. However, AMP will not attempt to centralize all models and data. Models and data may remain distributed on disparate computers in a variety of different formats. This approach eliminates most of the need to duplicate data or convert models to different operating systems.

AMP will provide the tools to interface disparate models and data to support assessments alternatives. In many instances, a cascading sequence of models and data are required. Models may execute on the local user's machine, a separate dedicated computer, a high-throughput distributed computing system or a commercial modeling services provider.

All three of the AMP toolboxes will provide capabilities specifically to enhance accessibility for a variety of needs and users. The Decision Toolbox will include a Tradeoff Tool that visually communicates tradeoffs between objectives and their uncertainties. The Model Toolbox will provide tools for defining an analysis sequence from a menu of models and data that can be used to assess alternatives and track the status of an assessment. The Data Toolbox will provide tools to visualize, organize, and understand spatial and tabular data from multiple disparate sources.

\subsubsection{Web accessible}

AMP ensures maximum access to the broadest audience by utilizing the Internet. The design of AMP anticipates continued improvement of the speed and capacity of the Internet. Along with these improvements are the continued concerns for Web security. While providing easy access is critical, AMP must also protect user privacy and data and model integrity. AMP will have a sophisticated security architecture that provides the ability to establish the appropriate level of security for various aspects of the system. This will allow developers of proprietary models and data to use the system without concern for loss of intellectual property.

\subsubsection{Drill-down}

AMP will provide the ability to drill-down through decisions, data and models. The Decision Toolbox will provide access to the rationale supporting decisions. With the Tradeoff Tool, AMP users will view multiple-objective tradeoffs, read the decision makers' rationale, and view the preferences of others that may have been involved in the decision. The Decision Toolbox will show users the models, key assumptions, and data that went into arriving at alternative outcomes. 
The Data Toolbox provides the primary capabilities for ensuring that users can track the entire sequence of assumptions, models and data on which an alternative's impact was estimated. AMP will not attempt to provide a centralized data warehouse for all data and models. AMP will ensure the adequacy of metadata to establish the complete pedigree of data and models that remain distributed across the Internet. The metadata allows the drill-down tool to move throughout the Internet to obtain the information on the data and models used in an assessment.

\subsubsection{Collaboration}

Access to the decision making process is important to stakeholders, experts and regulators, but can be crucial to the planners involved in making decisions. Focused collaboration can provide insights that save time and money during project assessment, design and implementation.

The Decision Toolbox will provide protected access for collaboration. With the Tradeoff Tool, AMP users will be able to view multiple-objective tradeoffs and view collaborator preferences (e.g. varied perceived costs of alternatives). This tool will be used to work with assessment or regulatory colleagues to evaluate data and models, or with stakeholders to identify important issues.

\subsubsection{Adaptability}

As with all technology-based approaches, AMP must adapt continuously and rapidly to new data, models, and needs or the system will soon be obsolete. AMP must also help planners to be adaptive in their management approach, giving them greater flexibility and responsiveness.

- AMP will incorporate a modular modeling design that allows new data and models to be integrated into analyses.

- AMP will support real-time assimilation of data by providing tools to automate the calibration process.

- AMP will provide tools required to support adaptive management.

- AMP will streamline environmental planning, allowing it to occur earlier in the planning process.

\subsubsection{Modular modeling}

The ability of environmental scientists to use advanced models in environmental planning is controlled by the ability to link disparate models running on disparate platforms. AMP does not attempt to transform various models to run on a common platform. Models can be run on the users platform or an independent platform depending on the requirements and availability of the model. AMP will ensure that the communication between the models (and their respective platforms) is adequate and self-documenting. By managing the messaging between the models, AMP will provide a robust approach for model management. 
Successfully linking models depends on the ability of measurements, response models, process models, and policy models to interact with each other across a variety of respective temporal and spatial scales. For example, precipitation is measured at one scale $\left(1 \mathrm{~cm}^{2}\right)$ while runoff is measured as streamflow at much larger scales $\left(10 \mathrm{~km}^{2}\right)$. Furthermore, policy decisions (e.g., reservoir operations or irrigation scheduling) depend on the process models, but concern different temporal and spatial scales issues $\left(500 \mathrm{~km}^{2}\right)$. However, the inherent difficulties in reconciling scales have limited the applicability of science-based process models to the needs of policy-driven agencies. The reliability of natural resources management policy depends on the ability of measurements, response models, process models, and policy models to interact with each other across the variety of temporal and spatial scales each represents.

Scaling approaches in environmental applications must include a thorough understanding of the processes involved. The Model Toolbox will provide tools called 'scale filters' to ensure that information communicated between models has been corrected to the appropriate spatial and/or temporal scale.

\subsubsection{Real-time data assimilation}

Many environmental assessments fail because of loss of credibility from not using the 'most current' data. Often new data requires model recalibration. Model calibration is a significant cost in any modeling effort. Automating the calibration process allows real time updating of data/models allowing scientists to focus efforts on improving models. Automating the calibration process allows advanced models to be employed without unacceptable delays in delivery of results. Auto-calibration procedures are also useful in uncertainty assessment because equally feasible (well calibrated) input and outputs are generated.

The Model Toolbox will contain an autocalibration procedure that explicitly considers multiple calibration objectives (e.g., magnitude and timing of streamflow peak).

\subsubsection{Adaptive management}

Adaptive management is an approach to environmental impact assessment and management that encourages the integration of environmental with economic and social understanding, and the notion of experimentation and response. One of the problems it was intended to address was that fundamental properties of a development or policy are firmly established early in the design process such that significant change can only be introduced in the process through conflict and extraordinary pressure from interest groups. Adaptive management was conceived as a systems approach building on ecology, physical sciences and systems sciences. It is experimental in nature, utilizing "trial-and-error" and allowing for both known and unknown information. Its goal is to embrace uncertainty, accepting partial understanding of processes, and producing policies and designs that are less sensitive to the unexpected.

AMP was developed to provide an information infrastructure consistent with the needs of Adaptive Management. By enhancing the communication of uncertainty and clearly articulating tradeoffs, AMP will provide such an infrastructure. 


\subsubsection{Streamlined Planning}

Using sophisticated models and tools that create an intuitive interface, AMP will allow environmental planning to occur earlier in the planning process. An earlier start and the ability to consider more alternatives gives planners and project designers more flexibility in how they identify and mitigate environmental issues. An earlier start also gives stakeholders a more meaningful voice in the process because more options are available to planners.

\subsection{Toolboxes}

AMP will be composed of three integrated toolboxes. Each toolbox was designed so that other tools can be easily added.

- The Decision Toolbox articulates tradeoffs and communicates uncertainty between various alternatives.

- The Model Toolbox integrates and shares models and process knowledge.

- The Data Toolbox provides web-based management of disparate data sources.

\subsubsection{Decision Toolbox}

AMP provides or supports the following features:

- Multiple objective tradeoff analysis,

- Web-based consultations with multiple users,

- Information "drill-down” capability,

- Tools for uncertainty analysis, and

- Tools for assessing the value of information.

- Ability to evaluate differing perceptions of costs and benefits

\subsubsection{Multiple objective tradeoff analysis}

Modern natural resource managers are constantly required to balance multiple, conflicting, incommensurate objectives in an environment characterized by high levels of uncertainty, varying data quality and availability, and competing models and approaches. The reliability of natural resources management policy and decisions depends on the ability of measurements, response models, process models, and policy models to interact with each other across the variety of temporal and spatial scales each represents. It also requires a cautious, probing, adaptive approach founded on fundamental economic principles, the success of which depends upon improved understanding, predictive accuracy, and iterative performance assessment. 
The Tradeoff Tool in the AMP Decision Toolbox will clearly articulate the tradeoff between multiple objectives.

\subsubsection{Web-based consultation}

The Tradeoff Tool will allow multiple users from multiple locations on the Internet to view the same data simultaneously. Participants may use the data to view the tradeoffs that interest them the most. The tradeoffs are positioned as axes on the graphs shown in the tradeoff tool. This provides for a distributed collaboration or negotiation process. All users can see the preferred alternative of the other users. While this doesn't guarantee consensus, it does help understand the other user's position and provide greater appreciation of the willingness of some compromise to find common ground.

\subsubsection{Drill-down capability}

AMP will provide reliable and rapid access to models, data, and the rationale that underpin decisions. Information can include metadata and prepared descriptions. The Data Toolbox will provide tools to create and track metadata.

\subsubsection{Uncertainty analysis}

It is not feasible to eliminate uncertainty. Reducing uncertainty in complex environmental planning issues can involve costly laboratory experiments, field experiments, and modeling analyses. The cost of reducing uncertainty can be justified, if the reduction in uncertainty would likely impact a significant management decision.

Because of its potential magnitude, uncertainty in environmental systems cannot be neglected in the decision process. Uncertainty in the outcomes predicted with models can be attributed to many sources of errors. These errors can propagate and compound throughout any analysis.

AMP will help to address uncertainty in the selection of assumptions, models, and data. The tradeoff tool allows the user to view the impact of various assumptions about key outcomes. If a large degree of sensitivity is found, the decision maker may decide to:

- Study the assumptions further,

- Select the alternatives without significant adverse impacts under a variety of assumptions, or

- Select an alternative that through careful monitoring is most likely to reduce similar decision uncertainty in the future.

When model or data uncertainty are an issue, AMP Model Toolbox and Data Toolbox will provide flexibility in using a variety of model and data combinations. More sophisticated models and data, or the selection of alternate approaches, can help to address challenges. 
Many natural resources assessments fail because of loss of credibility from not using the 'most current' data. Automating the calibration process allows advanced models to be employed without unacceptable delays in delivery of results. Auto-calibration procedures are also useful in uncertainty assessment because equally feasible (well calibrated) input and outputs are generated. The autocalibration procedure explicitly considers multiple calibration objectives (e.g., magnitude and timing of streamflow peak) using a Pareto genetic algorithm. The generalized calibration tool to be included in the AMP's Model Toolbox is ideal for distributed computing.

\subsubsection{Value of information assessment}

An important concept inherent in adaptive management is that of "value of information." The engineering definition of "value of information" is simply the ability of additional information to reduce uncertainty in design variables, thereby accruing cost savings from a less conservative (i.e., lower cost) design. The value of additional (new) information can be exploited in a number of ways. At one extreme is the use of new information merely as data in a purely statistical context (e.g., least squares analysis). This is the minimal use of new information. Each new piece of data simply increases the degrees of freedom of the statistical model by one, with only marginal impact on standard errors of estimation and minimal increase in understanding. At the other end of the spectrum, information can be interpreted in the context of formulated models that fully utilize a priori information in representing process knowledge. In this context, new information can contribute to reducing uncertainty of predictions on multiple fronts such as model formulation improvement, enhancing model calibration, and/or model validation.

One of the most powerful elements of the adaptive management approach is its reliance on "value of information" concepts in making investments for new information. The concept enables a decision maker to fully utilize available information to analyze alternative solutions with full consideration given to the level and sources of uncertainty in the analysis. If those uncertainties impose excessive conservatism and therefore cost burden on the preferred alternative(s), then a clear tradeoff can be established on the costs (direct and indirect) of collecting new information, and the resultant benefits. Costs can include the actual costs of data collection, management and analysis, as well as indirect costs such as the impacts of delaying action until new data are obtained.

Benefits can also be viewed as direct or indirect. Direct benefits include avoided costs associated with project failures plus the savings derived from reduced uncertainty and design factors of safety. Indirect benefits are achieved principally from the increased process knowledge gained from new information and extension of that knowledge to future management decisions in the same watershed or to other watersheds in the region.

The AMP Decision Toolbox will provide the necessary tools to support "value of information" assessments. It considers the value of information in reducing decision errors by identifying patterns and anomalies in objective estimates. 


\subsubsection{Model Toolbox}

The reliability of natural resources management policy depends on the ability of measurements, response models, process models, and policy models to interact with each other across the variety of temporal and spatial scales each represents.

\subsubsection{Modular distributed modeling system}

An analysis of environmental planning alternatives typically involves a sequence of models. For instance, a hydrology model provides input to a stream chemistry model, which in turn, provides input to a fisheries survival model. These individual models (or sockets) must be coordinated by AMP. The models may be operated on a variety of platforms and be coded in a variety of languages. While AMP is designed to utilize a variety of models (sockets) and to allow users to constantly bring new improved models, it does not require that these models be translated to a common language or modified to run on a common platform. The AMP Model Toolbox provides a generalized set of tools and protocols to transform the outputs into the inputs of the following model. AMP will ensure that data and models are applied consistently throughout any analysis.

The linking of models and data will be managed through tools from the Data Toolbox. The analysis is sequenced by aligning data, model inputs, and model outputs as described in the AMP metadata.

\subsubsection{High-throughput computing}

One of the key advantages of AMP is its ability to support the analysis of more alternatives than is currently possible. To achieve this improvement, AMP provides tools for high-throughput computing. High-throughput computing involves orchestrating a large number of networked computers. Even small organizations have a large number of underutilized computers within their local network. By providing access to these computers (during off hours or in place of screen savers), a significantly larger analysis throughput is possible. AMP Model Toolbox provides high-throughput computing tools that are designed to be robust and work across a wide variety of platforms.

\subsubsection{Analysis tracking}

Given the large, complex and generally distributed nature of the modular modeling activities, it is critical that AMP users have the ability to track the progress of their analyses. In a multiple user environment this also provides for some prioritization of model runs by the AMP administrator. This also helps to identify critical paths in the overall analysis process and may be the basis for securing additional resources (improved models, faster computers, larger bandwidth networks, etc.).

\subsubsection{Automated calibration tools}

Model calibration is a significant cost in modeling effort. Automating the calibration process allows real time updating of data/models allowing scientists to focus effort on analyzing results. 
Many natural resources assessments fail because of loss of credibility from not using the 'most current' data. Automating the calibration process allows advanced models to be employed without unacceptable delays in delivery of results. Auto-calibration procedures are also useful in uncertainty assessment because equally feasible (well calibrated) input and outputs are generated.

The AMP auto-calibration procedure explicitly considers multiple calibration objectives (e.g., magnitude and timing of streamflow peak) using a Pareto genetic algorithm. This generalized calibration tool is ideal for distributed computing.

\subsubsection{Linking physical and ecosystem models}

In addition to scale differences, fundamental structural differences between physical and ecosystem models significantly complicate their linkage. Whereas physical models rely on formulas defined over a continuous range, ecosystem models are based on categorical statements about the habitat. PNNL has recently advanced the use of a new set of tools utilizing fuzzy logic to manage some of these issues. The fuzzy logic deals explicitly with the ambiguity in the terms used in ecosystem models categorical statements. AMP will include FuzzyHab, a web-based tool for assisting the linkage of physical and ecological models.

\subsubsection{Data Toolbox}

AMP will provide tools to manage and visually analyze data from distributed sources.

\subsubsection{Visual analysis}

To understand spatial and temporal environmental data, the ability to generate spatial maps and temporal plots and/or spatial animations is critical. Maps with multiple layers of different data type allow the user to understand spatial and temporal relationships between the various data. Tabular data can also be made accessible and shown in its correct georeferenced position on a map. Maps can also provide portals to information such as Native American 'cultural stories'.

AMP does not attempt to replace or duplicate the considerable developments made recently in GIS technology. Instead, AMP leverages from the current set of tools (proprietary and government) to ensure that AMP users can access maps of complex spatial and temporal datasets directly through their web browsers. AMP provides the tools to merge, transform and filter environmental data from disparate sources into consistent and informative maps.

\subsubsection{Distributed data management}

Natural resources management involves a large amount (albeit often sparse) and diverse data. Each data set can be quite large, often involving spatial data, such as remote sensing data. Unfortunately, there is no consistent structure to the data so the data management system must be able to access and process large spatial data. 'Data' as used herein refers to actual field measurements, remote sensing, model input and output, and available information regarding the pedigree of each. 
A credible data management system is essential for successful natural resource planning. AMP will not attempt to build a single large database from the distributed databases. Instead, it strives to leave as much data as possible on their native platforms. AMP manages the metadata and provides the pipeline linking data and models together.

In order to link data to models, it is often necessary to fill in holes in the original database. AMP provides the capability to estimate the data values in such data gaps using robust tools such as artificial neural networks, to assimilate large amounts of data from the particular region considered and other similar regions, to establish patterns and similarities within the environment considered.

Another issue in linking models to data and models to models is the change in spatial and temporal scales required. AMP provides 'scale filters' to transform data to the correct scale. Some scaling processes can be generalized, for instance 'upscaling' spatial data can often be done by taking the average of the subscale values. 'Downscaling' is generally more complex and requires some process understanding on which to base the downscaling process. AMP does not attempt to provide a complete set of 'scale filters'. AMP only provides a small set of such filters, as examples of the protocols required to develop such filters. AMP does however provide tools to identify likely scale issues through tracking scale information in the data and model metadata. 

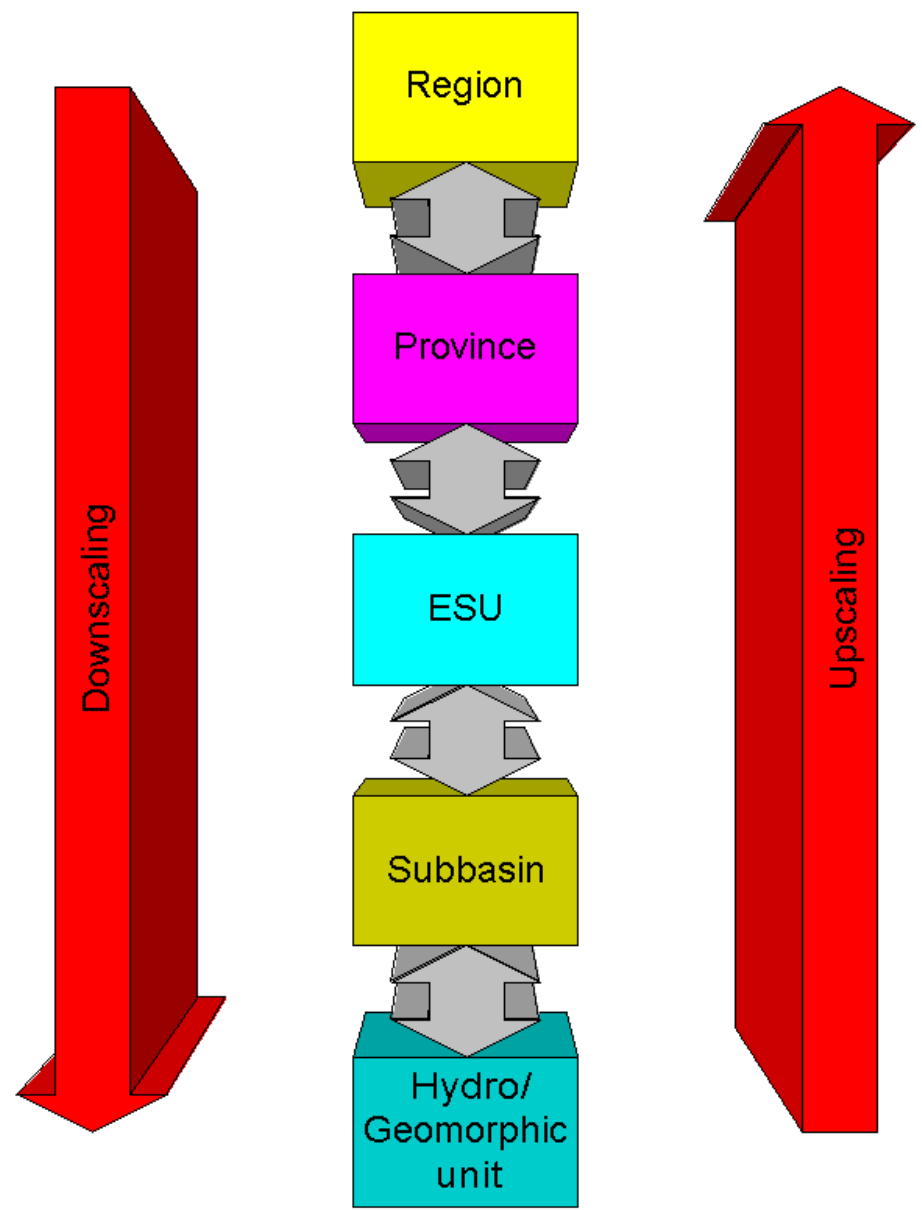

Figure 4. Supporting Regional and Local Planning

\section{Conclusions}

AMP is an information and analytic infrastructure (or platform) capable of supporting the planning and needs of natural resource managers in the Columbia River Basin. This Adaptive Management Platform (AMP) will provide the ability to defensibly, consistently and rapidly evaluate restoration policy and specific strategies at the subbasin scale relative to a basin-wide vision.

AMP will fully exploit available data, information and analytical methods (e.g., models) to establish comprehensive, science-based tradeoff relationships between various alternatives. AMP will support planners in making and defending decisions while simultaneously enabling stakeholders to understand and participate in the decision-making process. AMP will enable decision makers and planners to:

- Understand and communicate tradeoffs between different alternatives,

- Quantify real and expected productivity improvements from existing and planned projects, 
- Assess relative value of additional information,

- Utilize dramatically increasing volumes of data, and

- Utilize advanced models.

By providing the necessary information infrastructure in a nonproprietary platform, AMP will allow environmental planning to: start much earlier, be performed for significantly lower cost, consider alternatives more completely, utilize advanced models and data, and advance more rapidly toward implementation.

Significant improvements in Accountability, Accessibility, and Adaptability were identified as three critical requirements of AMP. To provide these requirements, integrated toolboxes (for decisions, models, and data) will include both software and hardware.

- Accountability - The primary goal of AMP is to support making decisions. To be useful, it must ensure that the tradeoffs between multiple objectives (of interest to both decision maker and stakeholders) are clearly articulated. AMP should also communicate the sources and magnitude of key uncertainties.

- Accessibility - AMP will provide rapid access to models, data, and the rationale that underpin decisions. These are growing requirements of natural resource management. The ability to drill-down through data and models ensures that decision makers are less likely to repeat decision errors.

- Adaptability - AMP must be able to rapidly assimilate new data and models to ensure that decisions are up-to-date with the most current information. Specific tools to automate the calibration process and to assimilate real-time data are requirements of the framework.

When deployed AMP will:

- Be decision-focused,

- Encompass basin, ecoprovince, and subbasin scales,

- Reflect integrated effects of management alternatives and natural variability on habitat conditions over long time-periods (e.g., climate variability),

- Address species of concern,

- Allow economic values to be placed on alternatives under consideration,

- Be widely accessible and facilitate regional collaboration, and

- Provide the foundation for sustainable adaptive management practices across the Columbia Basin. 


\section{Acknowledgement}

The authors gratefully acknowledge the support provided by the U.S. Environmental Protection Agency through Science to Achieve Results (STAR) Grant \#R827454 in preparing this document. 